\title{
Watermark Recovery from a Dual Layer Hologram with a Digital Camera
}

\author{
Anu Pramila*, Anja Keskinarkaus, Esa Rahtu, and Tapio Seppänen \\ Department of Electrical and Information Engineering, \\ University of Oulu, P.O. Box 4500, FIN 90014 University of Oulu \\ Tel.: +358-8-553-2797; Fax: +358-8-553-2534 \\ \{anu.pramila, anja.keskinarkaus, esa.rahtu, tapio.seppanen\}@ee.oulu.fi \\ http://www.cse.oulu.fi
}

\begin{abstract}
In this paper we present a method for reading a watermark from a dual layer hologram image with a digital camera. Here the base of the hologram out of which the hologram was designed is a binary image. The hologram obtained is placed on a planar surface and an image is captured from the hologram by setting up a light source and a digital camera on appropriate distances and angles relative to the hologram. The captured image is corrected from affine distortions and a saliency detector based segmentation is performed for the image. The watermark is read from the resulting reconstructed binary image. The obtained results show that the watermark can be recovered perfectly from a dual layer hologram with a proper setting of the camera and light sources.
\end{abstract}

Keywords: Digital watermarking, watermarking holograms, print-cam process.

\section{Introduction}

Holograms and watermarking have both been considered as safety measures against copying. In this paper, these two techniques are combined and a method for recovering a watermark from a dual layer hologram is introduced. This adds another level of security to the holograms.

Wang et al. 1] proposed a method for watermarking a dot matrix hologram. For embedding the watermark they used a modified halftoning technique exclusively designed for dot matrix color hologram. When extracting the watermark, the hologram was photographed by carefully selecting a high resolution digital camera and proper lighting conditions.

However, there are many hologram techniques available and here a dual layer hologram as shown in Fig. 1 was manufactured and used instead of a dot matrix hologram as in [1]. The hologram consists of a background image and foreground image layers. The frame and the pseudorandom pattern on the hologram background were considered only as artistic features.

\footnotetext{
* The financial support from the Academy of Finland and Graduate school of is gratefully acknowledged. Hologram by courtesy of Starcke Ltd.
} 

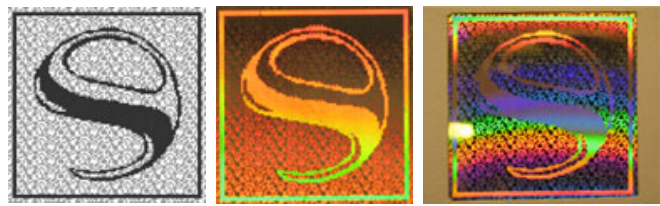

Fig. 1. a) A design for the hologram. b) The obtained hologram. c) The phase change in the hologram background and foreground.

When reading a watermark from a printed image with a digital camera, the watermark should be robust against 3 dimensional distortions, rotation, scaling, and translation 234 . In addition, there may be variations in the environment, such as lighting and reflections. The camera itself inflicts various attacks to the watermark, for example, JPEG-compression and lens distortions [56].

When reading a watermark from a hologram, the problems to be solved are increased. The hologram surface is often mirror like and the watermarked image may not be visible in all directions or with all light sources. Source and direction of the light affects the way the hologram is seen. Due to the rainbow effect on the surface of the hologram, the color and intensity changes across the watermarked image when the hologram is tilted relative to the light source. However, it can be assumed that the color varies in the background of the image in different phase to that of the foreground as illustrated in Fig. 1].

In this paper, based on the notion of locally different colors on the background and foreground, the hologram image is segmented and the watermark read. Original unwatermarked image is employed in the process. The segmentation method used in the process is based on a method by Rahtu et al. 9]. The segmentation process is explained with more detail in Section 2.2

Here the hologram is created from a binary image. Due to the reflective nature of the hologram, it is difficult to detect single separate pixels and thus pattern matching methods were used to modify the method by Tseng et al. [8]. The watermarking method is explained with more detail in Section 2.1. The experiments were made and the test set-up and results are shown in Chapter 3 .

\section{Methods}

In this chapter, the method applied is explained in detail. First the watermarking method which was applied is explained, then the correction of geometrical distortions and finally image segmentation algorithm is described.

\subsection{Watermarking Method}

Pan et al. [7] proposed a data hiding method for binary images. Tseng et al. 8] later identified some image quality issues and improved the method. In this paper 
we have modified the method by Tseng et al. by applying patten matching methods in order to better adapt the method for our application. Below is a review of the method by Tseng et al. and afterwards the modifications are explained.

In the methods by Pan et al. [7] and Tseng et al. 8] the main idea is to use a secret key and a weight matrix to protect the hidden data. It is shown that for each $m \times n$ block in the host binary image $I, r \leq\left\lfloor\log _{2}(m n+1)\right\rfloor-1$ bits of data can be embedded by changing at most 2 bits in the block.

In the following, $K$ is a key, a randomly selected binary matrix of size $m \times n$. $\mathrm{W}$ is a weight matrix which is an integer matrix of size $m \times n$. Here, $[W]_{i, j}$ denotes the element of $W$ at row $i$ and column $j$. W satisfies the condition that $\left\{[W]_{i, j} \mid i=1 \ldots m, j=1 \ldots n\right\}=\left\{1,2, \ldots, 2^{r+1}-1\right\}$ and each $2 \times 2$ sub-block contains at least one odd element. 8

Tseng et al. [8] aimed to improve the method by Pan et al. 7] by introducing a distance measure in order to ensure that the modified bit is adjacent to another bit with the same value. The distance matrix was calculated with

$$
[\operatorname{dist}(I)]_{i, j}=\min _{\forall x, y}\left\{\sqrt{|i-x|^{2}}+|j-y|^{2} \mid[I]_{i, j} \neq[I]_{x, y}\right\}
$$

where $[\operatorname{dist}(I)]_{i, j}$ is the distance from $[I]_{i, j}$ to the closest element $[I]_{x, y}$ such that the complement of $[I]_{i, j}$ is equal to $[I]_{x, y}$.

A bit stream $b_{1} b_{2} \ldots b_{r}$ is embedded into each non-black and non-blank host block $I_{i}$ by first computing following set for each $w=1 . .2^{r+1}-1$ :

$$
\begin{gathered}
T_{w}^{\prime}=\left\{(j, k) \mid\left[\left([W]_{j, k}=w\right) \wedge\left(\left[I_{i} \oplus K\right]_{i, j}=0\right) \wedge\left([\operatorname{dist}(I)]_{j^{\prime}, k^{\prime}} \leq \sqrt{2}\right)\right]\right. \\
\left.\vee\left[\left([W]_{j, k}=2^{r+1}-w\right) \wedge\left(\left[I_{i} \oplus K\right]_{i, j}=1\right) \wedge\left([\operatorname{dist}(I)]_{j^{\prime}, k^{\prime}} \leq \sqrt{2}\right)\right]\right\},
\end{gathered}
$$

where $[\operatorname{dist}(I)]_{j^{\prime}, k^{\prime}}$ is for the bit corresponding to $\left[I_{i}\right]_{i, j}$ in block $I_{i}$. $\oplus$ means bitwise exclusive or of two binary matrices. Here each bit has 8 neighbors and thus the distance $\leq \sqrt{2}$. 8 ]

Second, a weight difference is defined $(\otimes$ means pairwise multiplication of two matrices and $S U M$ means the sum of all elements in a matrix)

$$
d^{\prime} \equiv\left(b_{1} b_{2} \ldots b_{r} 0\right)-S U M\left(\left(I_{i} \oplus K\right) \otimes W\right)\left(\bmod 2^{r+1}\right) .
$$

If $d^{\prime}=0$, there is no need to change $I_{i}$. Otherwise [8]:

if(there exists an $h \in\left\{0,1, \ldots, 2^{r}-1\right\}$ such that $T_{h d^{\prime}}^{\prime} \neq \emptyset$ and $\left.T_{-(h-1) d^{\prime}}^{\prime} \neq \emptyset\right)$

Randomly pick an $\mathrm{h}$ which satisfies the above condition;

Randomly pick a $(\mathrm{j}, \mathrm{k}) \in T_{h d^{\prime}}^{\prime}$ and complement the bit $\left[I_{i}\right]_{j, k}$; else

Randomly pick a $(\mathrm{j}, \mathrm{k}) \in T_{-(h-1) d^{\prime}}^{\prime}$ and complement the bit $\left[I_{i}\right]_{j, k}$;

$$
\text { if }\left(S U M\left(\left(I_{i} \oplus K\right) \otimes W\right) \bmod 2=1\right)
$$

Keep $I_{i}$ intact;

else

Select a $(j, k)$ such that $[W]_{j, k}$ is odd and its corresponding $[\operatorname{dist}(I)]_{j^{\prime}, k^{\prime}}$

is the smallest and complement the bit $\left[I_{i}\right]_{j, k}$;

end

end 
If the resulting modified image block $I_{i}^{\prime}$ is completely black or blank, the data hiding is regarded as invalid. The same bit sequence $b_{1} b_{2} \ldots b_{r}$ is hidden to the next block if applicable. 8]

The receiver computes the hidden data from each block with $\left(S U M\left(\left(I_{i}^{\prime} \oplus\right.\right.\right.$ $K) \otimes W)) / 2$, if $I_{i}^{\prime}$ is not completely black or blank and $S U M\left(\left(I_{i}^{\prime} \oplus K\right) \otimes W\right)$ is even. Otherwise, $I_{i}^{\prime}$ contains no hidden information. 8

The method by Tseng et al. 8] guarantees that each modified bit is neighboring a bit that is equal to the new value of the modified bit. Authors focus on 8-neighborhood which, however, results in sporadic pixels such as in Fig. 2 .
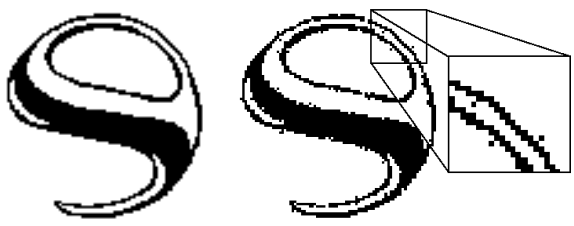

Fig. 2. Original image and a watermarked image with the method by Tseng et al.

In holograms, the shiny surface inflicts spreading of the pixels in the captured image. Therefore, sporadic pixels may be difficult to detect reliably. In order to minimize the possibility for sporadic pixels due to the watermarking method, we modify the method by Tseng et al. 8] by applying pattern matching methods to determine possible locations for the watermarked pixels. The watermark is embedded only in those predetermined locations on such a way that the reading method of the watermark is not affected. The patterns used are in Fig. 3.

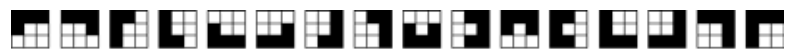

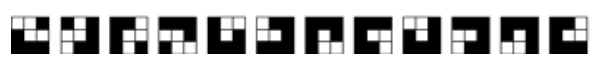

Fig. 3. Patterns used for watermark embedding

Each pixel neighborhood is compared with each of the patterns. If a mach is found the pixel is marked as a possible location for a watermark bit. Therefore $T_{w}^{\prime}$ becomes

$$
\begin{aligned}
T_{w}^{\prime \prime} & =\left\{(j, k) \mid\left[\left([W]_{j, k}=w\right) \wedge\left(\left[I_{i} \oplus K\right]_{i, j}=0\right)\right.\right. \\
& \left.\wedge\left([\operatorname{dist}(I)]_{j^{\prime}, k^{\prime}} \leq 1\right)\right] \vee\left[\left([W]_{j, k}=2^{r+1}-w\right)\right. \\
& \left.\left.\wedge\left(\left[I_{i} \oplus K\right]_{j, k}=1\right) \wedge\left([\operatorname{dist}(I)]_{j^{\prime}, k^{\prime}} \leq 1\right)\right] \wedge\left([M]_{j, k}=1\right)\right\}
\end{aligned}
$$

where $M$ is a matrix, containing obtained results of the pattern matching. The $\sqrt{2}$ is changed to 1 because we are interested only of 4 -neighborhood. The watermark is read as in the method by Tseng et al. [8] and the final watermarked image can be seen in Fig. 4 . 


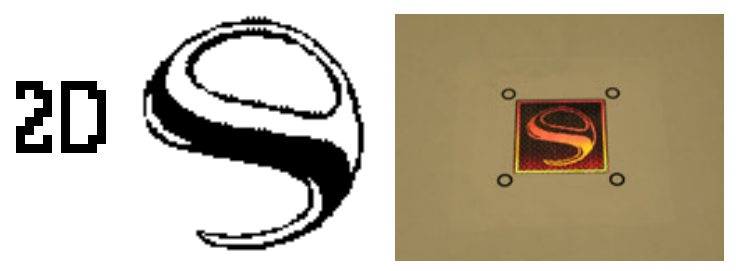

Fig. 4. a) Watermark message as an image. b) Watermarked binary image. c) Final hologram and synchronization markings.

\subsection{Correcting Distortions and Segmentation}

The binary watermarking method is very sensitive to geometrical distortions and as was noted in [10] accurate synchronization is required so that the binary image can be restored. Here a circular synchronization template, as illustrated in Fig. 4 is placed on each corner of the image and the user is prompted to select these templates from the captured image through a user interface before the captured image is processed and the watermark read. The exact locations of the circles are known and thus the distortions can be calculated and corrected with the following affine transformation.

$$
x^{\prime}=\frac{a_{1} x+b_{1} y+c_{1}}{a_{0} x+b_{0} y+1}, y^{\prime}=\frac{a_{2} x+b_{2} y+c_{2}}{a_{0} x+b_{0} y+1},
$$

where $\left(x^{\prime}, y^{\prime}\right)$ are the original picture positions, $(x, y)$ are the camera picture positions and $a, b$ and $c$ are coefficients calculated from the circle locations.

In order to recover the watermarked binary image, the captured and corrected hologram image is segmented. The segmentation is done for a significantly larger image than the original so that no information is lost. Here we used an image size five times larger than the original, the size of which was $94 \times 90$ pixels.

The original unwatermarked binary image is given to the segmentation algorithm as an initial assumption. However, because the color varies in the background of the hologram in different phase to that of the foreground, it is advantageous work with RGB color space instead of grayscale images. By experimenting, it was discovered that the segmentation algorithm operates best if the binary image is not copied to each of the channels but instead roughly divided among each of the channels by first calculating approximately which channels hold most of the information corresponding to the binary image. This is done by calculating for each channel $c$

$$
g_{i}(c)=\frac{\sum_{j}^{N} \sum_{k}^{L} U_{i}(c) B_{i}}{\sum_{j}^{N} \sum_{k}^{L} U_{i}(c)\left(1-B_{i}\right)},
$$

where $U_{i}$ is $i$ th block on the corrected and captured image $U, B$ is the original binary image and $M$ and $L$ the size of the image block. It is assumed that the 
colors run horizontally on the image and thus a horizontal block with width of that of the image and height of 30 was used here. Now, the binary template $B^{\prime}$ is calculated with

$$
\begin{aligned}
& B_{i}^{\prime}\left(c_{1}\right)=B_{i} \\
& B_{i}^{\prime}\left(c_{2}\right)=B_{i} \text { if } g_{i}\left(c_{1}\right)-g_{i}\left(c_{2}\right)<0.4,
\end{aligned}
$$

where $c_{1}$ is the channel where $g_{i}$ is the largest and $c_{2}$ is the channel where $g_{i}$ was the second largest.

The segmentation is based on a method by Rahtu et al. 9] and detects visually salient areas. The method applies a sliding window approach where a window is moved across the image and the saliency of a point in the window is estimated by determining the conditional probability of a pixel to be realized from the distribution estimated inside the window compared to the distribution of the surrounding area.

In the following, a rectangular window $U_{w}^{\prime}$ is divided into an inner kernel and an outer border, based here on the binary template. $F$ contain feature values, i.e. here we used the RGB values as features. Two hypotheses are defined, $H_{0}$ : point $\mathrm{x}$ is not salient, and $H_{1}$ : point $\mathrm{x}$ is salient. Corresponding priori probabilities are $P\left(H_{1}\right) P\left(H_{0}\right)=1-P\left(H_{1}\right)$. The initial assumption is that $H_{1}$ is valid for points in the kernel and $H_{0}$ is valid for points in the border. The conditional feature distributions $p\left(F(x) \mid H_{1}\right)$ and $p\left(F(x) \mid H_{0}\right)$ are estimated from the feature values $F$ in kernel and border. Then with Bayes theorem [9]

$$
P\left(H_{1} \mid F(x)\right)=\frac{p\left(F(x) \mid H_{1}\right) P\left(H_{1}\right)}{p\left(F(x) \mid H_{0}\right) P\left(H_{0}\right)+p\left(F(x) \mid H_{1}\right) P\left(H_{1}\right)}, x \in \Re^{2} .
$$

The saliency measure $S(x)$ is thus defined as $S(x)=P\left(H_{1} \mid F(x)\right)$.

A window $U_{w}^{\prime}(i)$ is slid over the image $U^{\prime}$ using a step $s_{w}$ and measure $S_{i}(x)$ is calculated at each window position $i$. The step $s_{w}$ is defined such that windows do overlap and the final saliency value of a pixel is defined as maximum 9]

$$
S(x)=\max _{j}\left\{S_{j}(x) \mid x \in U_{w}^{\prime}(j)\right\}
$$

The final salient objects were segmented from the background by thresholding $S(x)$. The threshold corresponds to the lowest probability which is allowed for a salient pixel to have for hypotheses $H_{1}$. Further, all 4-connected sets that cover less than 0.1 percent of the image area were removed and a morphological closing was performed with disk of radius $0.01 * \min \left\{w_{U^{\prime}}, h_{U^{\prime}}\right\}$ where $w_{U^{\prime}}$ and $h_{U^{\prime}}$ are the image width and height respectively. the prior probability was set as $P\left(H_{1}\right)=0.25$. For RGB image, the measure was calculated independently for each channel and maximum was taken as the final saliency value. 9]

In a hologram image the color varies across the image depending on the alignment of the hologram in relation to the light source. A red area might be background in one part of the image and foreground in the other part of the image. The sliding window size is here selected experimentally to be smaller than assumed color variation but big enough to lead to a reliable segmentation. It is 
assumed that the light source is aligned horizontally to the hologram and thus the window size was selected here such that $w_{U_{w}^{\prime}}=150$ and $h_{U_{w}^{\prime}}=50$ with step size $s_{U_{w}^{\prime}}=20$, for image size $w_{U^{\prime}}=470$ and $h_{U^{\prime}}=450$. However, due to the properties of the binary image, 8-connected sets that cover less than 0.01 percent of the image area were removed instead of 4-connected sets.

The segmentation method does not take into account the fact that the pixel size in the corrected image is a multiple of the pixel size in the original image. Therefore, while segmenting just before thresholding, the image is divided into blocks of size $a \times a$, where $a$ is the multiple. All the pixels in a block are given the mean value of the center pixels of the block. The thresholded and segmented binary image is then scaled to its original size $94 \times 90$ and the watermark is read.

\section{Experiments}

Due to the high cost involved in hologram manufacturing, we were restricted in using only one hologram. The test setting is depicted in Fig. 5. The hologram was placed on a plane, the angle of which could be changed. A camera (Canon G7, $10 \mathrm{MP}$ ) was placed on a tripod and set in front of the hologram. The pedestal was set initially $10 \mathrm{~cm}$ away from the hologram plane. The test were conducted by varying the angle of the hologram plane and directing the camera accordingly as well as varying the camera pedestal distance from $10 \mathrm{~cm}$ to $15 \mathrm{~cm}$ and $20 \mathrm{~cm}$.

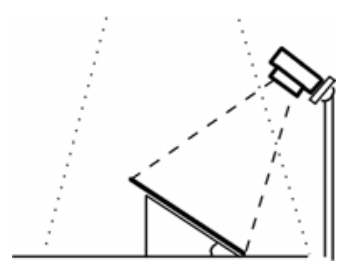

Fig. 5. The test setting. The light arrives from above the hologram which is set on a plane at an angle. The camera is directed towards the hologram.

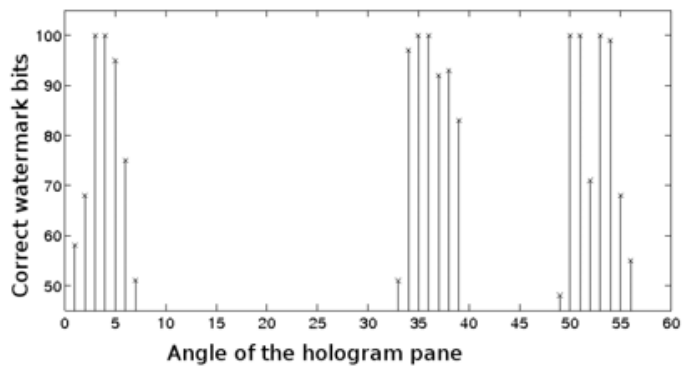

Fig. 6. Effect of changing the angle of the hologram plane 
The experiments were conducted by first holding the camera still and turning the hologram plane from 0 to 59 degrees, a degree at a time. The results were collected to Fig. 6 in which y-axis shows the amount of correct watermark message bits relative to the angle, 100 being the maximum. It can be seen from the image, that there are three locations at which the watermark was fully extracted and the segmentation successful. At these locations the watermarked image was lit fully, and the color and intensity of the background were locally different relative to the foreground. At the other locations, the watermark recovery is not possible due to the hologram properties; The logo is not fully lit and properly visible. No error correction coding was applied.

The results in the three locations are further illustrated in Fig. 7 and Fig. 8]a) and b). In Fig. 7 are the original image, corrected image, segmented image and the obtained watermark message respectively. In the Fig. 8 the original captured images are left out.

Next, the experiments were conducted by moving the camera away from the hologram. The angle was incremented from 49 to 59 degrees and the camera pedestal was moved from $10 \mathrm{~cm}$ to $15 \mathrm{~cm}$ and $20 \mathrm{~cm}$. The height of the pedestal was increased accordingly so that the view to the hologram stayed the same. The results are illustrated in Fig. 7 and Fig. 9 a) and b).

Together the experimental results show that the most important factor in watermark recovery reliability is not distance of the camera but the angle of the hologram relative to the camera and light source. Perfect recovery of the watermark was attained with certain angles of the hologram and correct photographing set-up.

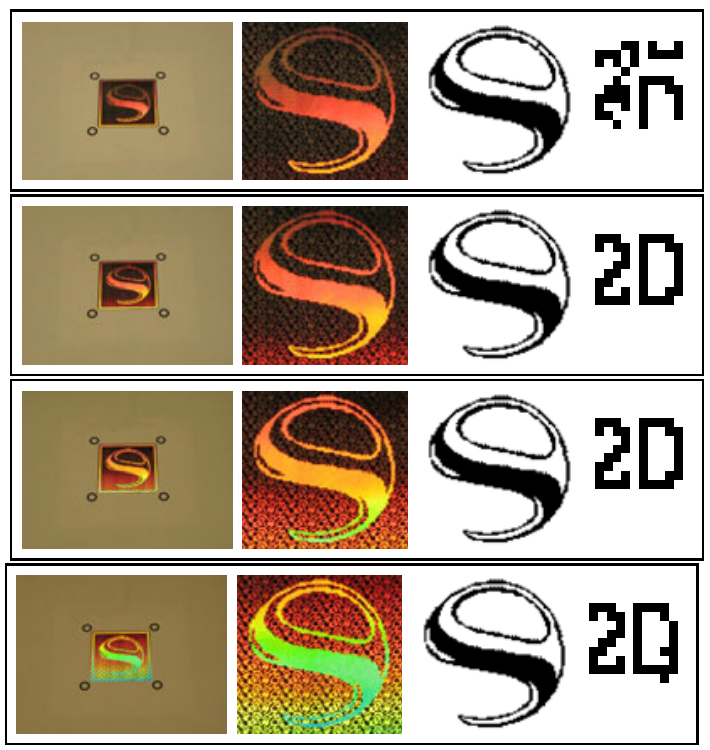

Fig. 7. Captured images with angles 2 to 5 and with distance $10 \mathrm{~cm}$ 

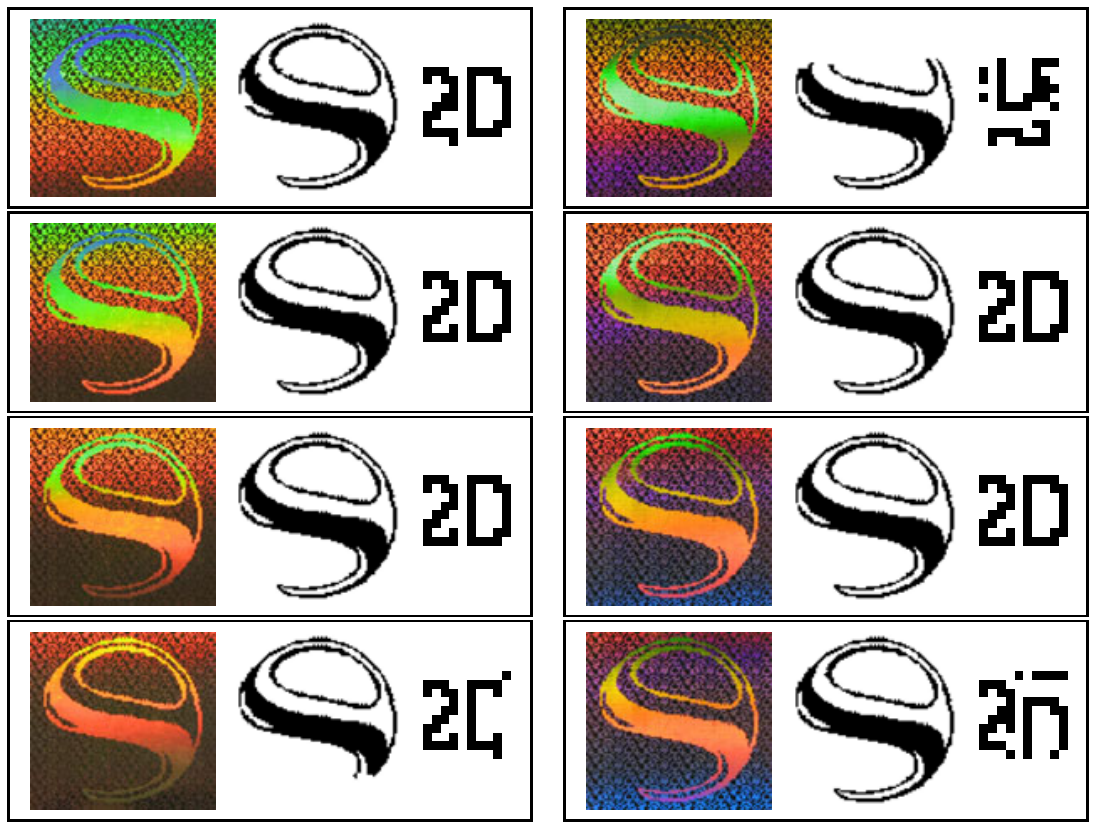

Fig. 8. Captured images with distance $10 \mathrm{~cm}$ and with angles a) 34 to 37 and b) 49 to 52
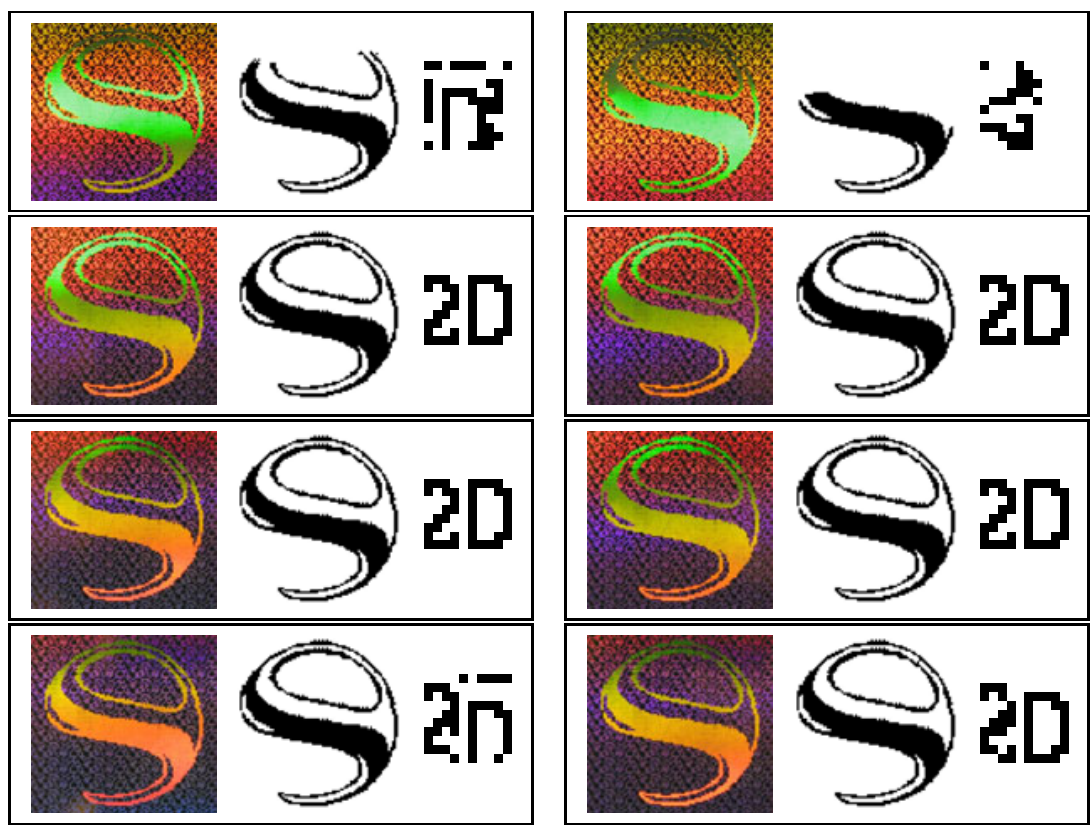

Fig. 9. Captured images with angles 49 to 52 and with distance a ) $15 \mathrm{~cm} \mathrm{~b}) 20 \mathrm{~cm}$ 


\section{Conclusion}

A method for reading a watermark from a dual layer hologram with a digital camera is presented in this paper. With correct set-up, the watermark was reconstructed perfectly. The recovery of the watermark from a hologram requires robustness against 3D distortions, color variations and reflections. The binary image needs to be reconstructed perfectly pixel by pixel in order to be able to read the watermark. The method was based on inversion of the distortions by applying synchronization markings around the image and segmenting the corrected image. In the future work, the aim is to remove the synchronization markings as well as take better into account the unique properties of the hologram.

\section{References}

1. Wang, H.-C., Wang, W.-C.: Data hiding in a hologram by modified digital halftoning techniques. In: Khosla, R., Howlett, R.J., Jain, L.C. (eds.) KES 2005. LNCS (LNAI), vol. 3683, pp. 1086-1092. Springer, Heidelberg (2005)

2. Lin, C.Y., Chang, S.F.: Distortion modeling and invariant extraction for digital image print-and-scan process. In: International Symposium on Multimedia Information Processing Taiwan (1999)

3. He, D., Sun, Q.: A practical print-scan resilient watermarking scheme. In: IEEE International Conference on Image Processing (ICIP), vol. 1, pp. I-257-I-260 (2005)

4. Solanki, K., Madhow, U., Manjunath, B.S., Chandrasekaran, S.: Estimating and Undoing Rotation for Print-scan Resilient Data Hiding. In: IEEE International Conference on Image Processing (ICIP), vol. 1, pp. 39-42 (2004)

5. Perry, B., MacIntosh, B., Cushman, D.: Digimarc MediaBridge - The birth of a consumer product, from concept to commercial application. In: Proc. of SPIE Security and Watermarking of Multimedia Contents IV, San Jose, CA, USA, vol. 4675, pp. 118-123 (2002)

6. Stach, J., Brundage, T.J., Hannigan, B.T., Bradley, B.A., Kirk, T., Brunk, H.: On the use of web cameras for watermark detection. In: Proc. of SPIE Security and Watermarking of Multimedia Contents IV, San Jose, CA, USA, vol. 4675, pp. 611-620 (2002)

7. Pan, H.-K., Chen, Y.-Y., Tseng, Y.-C.: A secure data hiding scheme for two-color images. In: Fifth IEEE Symposium on Computers and Communications, pp. 750 755. IEEE Press, Los Alamitos (2000)

8. Tseng, Y.-C., Pan, H.-K.: Secure and invisible data hiding in 2-color images. In: 10th Annual Joint Conference of the IEEE Computer and Communications Societies, vol. 2, pp. 887-896. IEEE Press, Los Alamitos (2001)

9. Rahtu, E., Heikkilä, J.: A simple and efficient saliency detector for background subtraction. In: IEEE Intl. Workshop on Visual Surveillance, Kyoto, Japan, pp. 1137-1144 (2009)

10. Pramila, A., Keskinarkaus, A., Seppänen, T.: Reading watermarks from printed binary images with a camera phone. In: Ho, A.T.S., Shi, Y.Q., Kim, H.J., Barni, M. (eds.) IWDW 2009. LNCS, vol. 5703, pp. 227-240. Springer, Heidelberg (2009) 